\title{
A Test Cases Library for Methods Locating the Sources of Sustained Oscillations
}

\author{
Slava Maslennikov ${ }^{1}$, Senior Member, IEEE, Bin Wang ${ }^{2}$, Student Member, IEEE, Qiang "Frankie" Zhang", \\ Member, IEEE, Feng Ma ${ }^{1}$, Member, IEEE, Xiaochuan Luo ${ }^{1}$, Senior Member, IEEE, Kai Sun ${ }^{2}$, Senior Member, \\ IEEE and Eugene Litvinov ${ }^{1}$, Fellow, IEEE \\ ${ }^{1}$ ISO New England \\ Holyoke, MA \\ \{smaslennikov, qzhang, fma, xluo, elitvinov\}@iso-ne.com \\ ${ }^{2}$ University of Tennessee \\ Knoxville, TN \\ \{bwang, kaisun\}@utk.edu
}

\begin{abstract}
A test cases library is created to provide a common foundation to test methods for locating the source of a poorly damped or forced oscillation by using wide-area measurements. The library is built on a simplified WECC 179-bus system and includes both poorly damped and forced oscillation cases. Three source location methods were fully tested using the library, and two selected cases for each method are presented in this paper to demonstrate the advantages and the disadvantages of each method. This library would help validate, evaluate and improve methods locating the oscillation sources in power systems.
\end{abstract}

Index Terms - Test cases library, source location method, forced oscillation, natural oscillation, sustained oscillation, poorly damped oscillation, negative damping.

\section{INTRODUCTION}

Massive deployment of Phasor Measurement Units (PMUs) in power systems has enabled the possibility to clearly monitor the actual dynamic behaviors of the system. The PMU measurements have helped detect multiple instances of poorly damped oscillations with amplitudes up to hundreds of MWs peak-to-peak in a wide frequency range from $0.02 \mathrm{~Hz}$ to $5-20 \mathrm{~Hz}$. For example, one of the oscillation events with unknown source of oscillations captured by ISO New England has a frequency of $0.12 \mathrm{~Hz}$ with $100 \mathrm{MW}$ peak-to-peak amplitude [1]. To help system operators better understand and manage these phenomena, there is a demand for tools that can reliably and accurately locate the source of oscillations [2]-[3].

High amplitude, long lasting, poorly damped oscillations, regardless of their types, represent a risk to stability and security in real-time operations; therefore such oscillations should be mitigated as soon as possible. The first step is to find the location of the source that has bad damping or produces forced oscillations. Effective remedial actions can

This work was supported by ISO New England and the CURENT Engineering Research Center.

S. Maslennikov, Q. Zhang, F. Ma, X. Luo and E. Litvinov are with ISO New England, Holyoke, MA, USA, and B. Wang and K. Sun are with the University of Tennessee, Knoxville, TN, USA. only be taken when the originating source is identified. Unfortunately, traditional modeling approach practically cannot help here because the location and nature of the source are typically unknown therefore cannot be accurately modeled. A measurement based location method is more practical when analyzing these real-life oscillations. With PMU measurements collected from different points in the system and providing sufficient observability, the model deficiency issue can be circumvented.

Locating the source usually means finding the power plant or the substation (if the source of oscillation is load or HVDC) where the oscillation originates. If PMU measurements are also available on individual generators within the source power plant, the location method should identify the source generator as well. However, tracing the source down to a specific module or a piece of hardware is beyond the scope of this library, because it requires internal measurements which are usually not available.

Locating the oscillation source is not a trivial task, particularly for inter-area oscillations. Research community has proposed several methods; however, few of them have yet been shown as a robust and efficient tool that could function in different scenarios [4]-[14]. As new methods are constantly being developed, how to test their effectiveness becomes a concern. It is clear that PMU data from real-life events cannot be used unless the actual source of the oscillation is known. Even if the actual source of a certain oscillation event is known and can be used for validation, the applicability of these methods to other situations still remains unclear.

In order to overcome this hurdle, a library of simulated test cases is developed. The library includes intentionally designed poorly damped oscillations and forced oscillations in the test system. Any source location method may be tested using these cases to verify its capabilities and limitations before applied to real events captured by PMU measurements. This paper first provides an overview on the development of this library and then presents some illustrative results of applying three 
existing source location methods with cases selected from the library. The results demonstrate the advantage and disadvantage of each method under test. Conclusions are given based on the analysis of the test results.

\section{TEST CASES LIBRARY}

\section{A. Overview of the test cases library}

The library is built based on a reduced WECC 179-bus, 29-machine system. The one-line diagram of the base case is shown in Fig.1. In the base case: all generators are represented as the GENCLS model from Siemens PTI PSS/E, i.e. a classical second-order differential model reflecting the motion of the rotor; damping parameter D for all generators are set to 4; all loads are modeled as constant MVA. Note: (i) generator inertia data were recreated reflecting the dynamics of interests in the Western Interconnection system and does not match exactly the actual system parameters; (ii) damping parameter D for each generator was artificially created such that the natural modes not of interests are reasonably damped.

PMU measurements are created as output from 40-second time-domain simulations in TSAT software by Powertech Labs Inc., where the integration step is 0.25 cycle and the output sampling rate is $30 \mathrm{~Hz}$. Duration of simulation and PMU sampling rate here are sufficient to observe the electromechanical oscillation in the range of $0.1-15 \mathrm{~Hz}$.

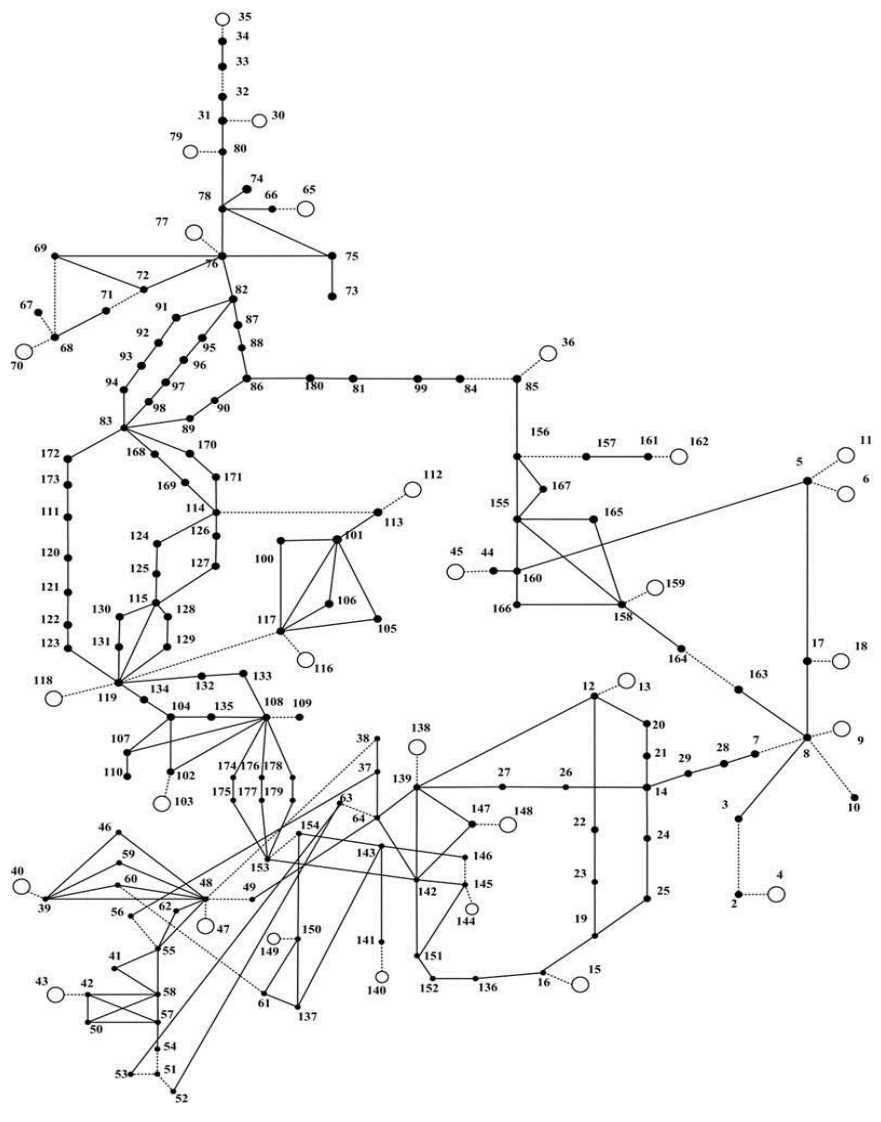

Fig. 1. WECC 179-bus system

Simulated PMU measurements provide full observability of the system including voltage magnitude and phase of all buses, current magnitude and phase of all branches from both sides, rotor speed and angle of all generators. Providing full observability of the system is important since it does not restrict signals being used, including generator rotor angle and speed. Signal availability may be a practical issue; however, if a certain location method is proven to be effective, requesting these signals will be justified.

Two types of oscillations are modeled in the library: poorly damped oscillations and forced oscillations. The poorly damped oscillation cases include a set of situations combining the following scenarios: a single or multiple sources, local modes or inter-area modes or a combination of both. The same base power flow is used for all test cases. Desired varieties of oscillation properties for undamped natural modes are generated by modifying damping coefficient $\mathrm{D}$ of certain generators and by applying faults at specific locations.

The forced oscillation cases include situations where the injected oscillation signal has a harmonic or a rectangular periodic shape. Each injected harmonic signal is tuned to have a frequency that (a) nearly equals to a frequency of a local or an inter-area mode and thus creating a resonance; (b) is close but above or below the frequency of a natural mode; (c) is between two local or inter-area modes. The non-harmonic periodic signals are designed so that each has a spectrum whose harmonics contain high energies and could coincide with an inter-area or a local natural modes. When modeling forced oscillations, the source generator is modeled with the GENROU model and an excitation system with typical parameters. Note that all GENROU models used in different cases in this library share the same set of parameters except for the inertia. A periodic disturbance is then modeled as a time-domain injection into the reference point of the excitation system. This is done by using a User Defined Model in the TSAT software.

All scenarios are designed after detailed modal analyses using the SSAT software by Powertech Labs Inc. Modal analyses provide the information about the natural modes (damping and frequency), mode shapes (right eigenvectors) and excitability of the modes (left eigenvectors). For a specific mode, the magnitudes and phases of elements in its right eigenvector associated with states of rotor angles indicate the observability and the grouping of generators for that mode, while the magnitude of each element in its left eigenvector indicate how large that mode will be excited when a fault is added to the location associated with that element.

SSAT software can also calculate the sensitivity of the real parts of the eigenvalues with respect to the damping coefficient $\mathrm{D}$ of each generator. This mode-damping-D sensitivity matrix has enabled the system behavior tuning through changing the parameter $\mathrm{D}$.

\section{B. Introduction of test cases}

All test cases are created on the modified WECC 179-bus power system. Detailed case descriptions, simulation results (to mimic PMU measurements) and the system models are available for the public to download at [15].

The first release of the library consists of 21 oscillation cases: 9 poorly damped/undamped electromechanical oscillation cases and 12 forced oscillation cases. More cases 
may be added in the future as our understandings of the problem progress.

\section{1) Poorly damped/undamped oscillation cases}

TABLE I summarizes the 9 cases in this category. In each case, any deviations of the damping coefficient $\mathrm{D}$ from the default value, i.e. 4, are shown in the second column of TABLE I, "PD" means poorly damped. For simplicity, it is used here to represent cases with poorly damped or undamped oscillations. The sources of the oscillations are the generators with negative $\mathrm{D}$ values. The detailed process of generating a poorly damped oscillation case is demonstrated using the case PD_1 in the following.

TABLE I SUMMARY OF POORLY DAMPED OSCILLATION CASES

\begin{tabular}{|c|c|c|c|c|c|c|}
\hline $\begin{array}{c}\text { Case } \\
\#\end{array}$ & D & $\begin{array}{c}\text { Freq } \\
(\mathrm{Hz})\end{array}$ & $\begin{array}{c}\text { Dampin } \\
\text { g ratio }\end{array}$ & $\begin{array}{c}\text { Source } \\
\text { bus }\end{array}$ & $\begin{array}{c}\text { Fault } \\
\text { bus }\end{array}$ & Description \\
\hline PD_1 & $\begin{array}{l}\mathrm{D} 45=-2 \\
\mathrm{D} 159=1\end{array}$ & 1.41 & $0.01 \%$ & 45 & 159 & $\begin{array}{l}1 \text { source } \\
1 \text { local mode }\end{array}$ \\
\hline PD_2 & $\begin{array}{l}\mathrm{D} 35=0.5 \\
\mathrm{D} 65=-1.5\end{array}$ & 0.37 & $0.02 \%$ & 65 & 79 & \begin{tabular}{|l}
1 source \\
1 inter-area mode
\end{tabular} \\
\hline PD_3 & $\begin{array}{l}\text { D6 }=2 \\
\text { D11=-6 }\end{array}$ & $\begin{array}{l}0.46 \\
0.70 \\
1.63\end{array}$ & $\begin{array}{l}2.22 \% \\
1.15 \% \\
-0.54 \%\end{array}$ & 11 & 30 & $\begin{array}{l}1 \text { source, } \\
1 \text { unstable local mode and } \\
2 \text { poorly damped inter- } \\
\text { area modes }\end{array}$ \\
\hline PD_4 & $\begin{array}{l}\text { D6 }=5 \\
\text { D11 }=-9\end{array}$ & $\begin{array}{l}0.46 \\
0.70 \\
1.63\end{array}$ & $\begin{array}{l}0.68 \% \\
-0.58 \% \\
0.54 \%\end{array}$ & 11 & 6 & $\begin{array}{l}1 \text { source } \\
1 \text { unstable inter-area mode } \\
\text { and } 2 \text { poorly damped local } \\
\text { and inter-area modes }\end{array}$ \\
\hline PD_5 & $\begin{array}{l}\text { D6 }=3 \\
\text { D11 }=-8\end{array}$ & $\begin{array}{l}0.46 \\
0.70 \\
1.63\end{array}$ & $\begin{array}{l}0.69 \% \\
-0.19 \% \\
-0.48 \%\end{array}$ & 11 & 30 & $\begin{array}{l}1 \text { source } \\
2 \text { unstable local and inter- } \\
\text { area modes and } 1 \text { poorly } \\
\text { damped inter-area mode }\end{array}$ \\
\hline PD_6 & \begin{tabular}{|l}
$\mathrm{D} 45=-2$ \\
$\mathrm{D} 159=-0.5$
\end{tabular} & 1.41 & $-0.93 \%$ & $\begin{array}{l}45 \& \\
159\end{array}$ & 159 & $\begin{array}{l}2 \text { sources with } \\
\text { comparable contribution } \\
\text { into a single unstable local } \\
\text { mode }\end{array}$ \\
\hline PD_7 & $\begin{array}{l}\mathrm{D} 45=-0.5 \\
\mathrm{D} 159=-0.5\end{array}$ & 1.41 & $-0.40 \%$ & $\begin{array}{l}45 \& \\
159\end{array}$ & 159 & $\begin{array}{l}2 \text { sources with different } \\
\text { contributions into a single } \\
\text { unstable local mode }\end{array}$ \\
\hline PD_8 & $\begin{array}{l}\text { D45 }=-2.5 \\
\text { D159 }=1 \\
\text { D36 }=-1\end{array}$ & $\begin{array}{l}1.27 \\
1.41\end{array}$ & $\begin{array}{l}-1.06 \% \\
-0.22 \%\end{array}$ & $\begin{array}{c}45 \& \\
36\end{array}$ & 159 & $\begin{array}{l}2 \text { sources } \\
2 \text { unstable local modes }\end{array}$ \\
\hline PD_9 & $\mathrm{D} 11=-10$ & $\begin{array}{l}0.46 \\
0.69 \\
1.63\end{array}$ & $\begin{array}{l}-0.86 \% \\
-1.81 \% \\
-0.40 \%\end{array}$ & 11 & 79 & $\begin{array}{l}1 \text { source } \\
3 \text { unstable modes }\end{array}$ \\
\hline
\end{tabular}

In the case PD_1, the parameter D for generators 45 and 159 are set to -2 and 1 , respectively, making the generator 45 a source. Small signal analysis shows that the local mode at $1.41 \mathrm{~Hz}$ has a low damping ratio at $0.01 \%$ and disturbance at bus 159 has a large excitability for this mode. Thus, in the time-domain simulation, a three-phase short circuit is applied on bus 159 at $0.5 \mathrm{~s}$ and cleared after $0.05 \mathrm{~s}$ to significantly excite the $1.41 \mathrm{~Hz}$ mode. Angle trajectories of generators 45 and 159 are shown in Fig.2. This case features a larger observability of the $1.41 \mathrm{~Hz}$ mode at bus 159 than that at the bus 45 . So the disturbance excites the maximal amplitude of rotor angle oscillations at generator 159 while the real source of the oscillation is actually located at generator 45 . This case is designed to challenge a common misconception that the source should have the largest amplitude, and the source location methods based on maximum oscillation amplitude.

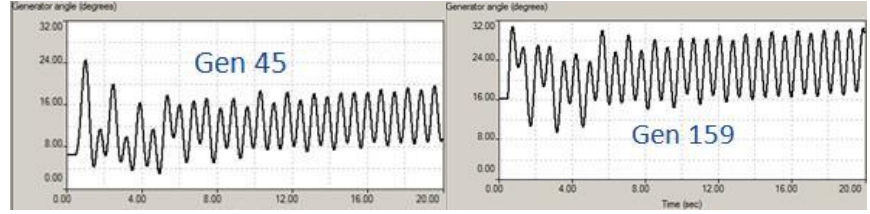

Fig. 2. Angle trajectories of generators 45 and 159

2) Forced oscillation cases

TABLE II summarizes the 12 cases in this category. In each forced oscillation case, all generators are modeled as classical model with $\mathrm{D}=4$ except for the source generator which is modeled as a round rotor generator with a periodic signal injected into its excitation system. The presence of the excitation system at one generator only slightly changes the signatures of the oscillations, so the same modal analysis is still applicable to the forced oscillation cases.

TABLE II SUMMARY OF FORCED OSCILLATION CASES

\begin{tabular}{|c|c|c|c|l|}
\hline $\begin{array}{c}\text { Case } \\
\#\end{array}$ & $\begin{array}{c}\text { Type of } \\
\text { injected } \\
\text { signal }\end{array}$ & $\begin{array}{c}\text { Freq of 1st } \\
\text { harmonic } \\
\text { (Hz) }\end{array}$ & $\begin{array}{c}\text { Source } \\
\text { location }\end{array}$ & \multicolumn{1}{|c|}{ Description } \\
\hline F_1 & Sinusoidal & 0.86 & 4 & $\begin{array}{l}\text { Resonance with local 0.86Hz } \\
\text { mode }\end{array}$ \\
\hline F_2 & Sinusoidal & 0.86 & 79 & $\begin{array}{l}\text { Resonance with local 0.86Hz } \\
\text { mode }\end{array}$ \\
\hline F_3 & Sinusoidal & 0.37 & 77 & $\begin{array}{l}\text { Resonance with inter-area } \\
\text { 0.37Hz mode }\end{array}$ \\
\hline F_4_1 & Sinusoidal & 0.81 & 79 & $\begin{array}{l}\text { Forcing frequency is below } \\
\text { natural 0.84Hz mode }\end{array}$ \\
\hline F_4_2 & Sinusoidal & 0.85 & 79 & $\begin{array}{l}\text { Forcing frequency is } \\
\text { between natural 0.84Hz and } \\
\text { 0.86Hz modes }\end{array}$ \\
\hline F_4_3 & Sinusoidal & 0.89 & 79 & $\begin{array}{l}\text { Forcing frequency is higher } \\
\text { than natural 0.86Hz mode }\end{array}$ \\
\hline F_5_1 & Sinusoidal & 0.42 & 79 & $\begin{array}{l}\text { Forcing frequency is below } \\
\text { natural 0.44Hz inter-area } \\
\text { mode }\end{array}$ \\
\hline F_5_2 & Sinusoidal & 0.46 & 79 & $\begin{array}{l}\text { Forcing frequency is } \\
\text { between natural 0.44Hz and } \\
\text { 0.47Hz inter-area modes }\end{array}$ \\
\hline F_5_3 & Sinusoidal & 0.50 & 79 & $\begin{array}{l}\text { Forcing frequency is higher } \\
\text { than natural 0.47Hz inter- } \\
\text { area mode }\end{array}$ \\
\hline F_6_1 & $\begin{array}{c}\text { Periodic, } \\
\text { rectangular }\end{array}$ & 0.1 & 79 & $\begin{array}{l}\text { Spectra of forced harmonics } \\
\text { consist of 0.1Hz, 0.3Hz, } \\
\text { 0.5Hz, 0.7Hz, etc modes }\end{array}$ \\
\hline F_6_2 & $\begin{array}{l}\text { Periodic, } \\
\text { rectangular }\end{array}$ & 0.2 & 79 & $\begin{array}{l}\text { Spectra of forced harmonics } \\
\text { consist of 0.2Hz, 0.6Hz, } \\
\text { lHz, 1.4Hz, etc modes }\end{array}$ \\
\hline $\begin{array}{l}\text { Feriodic, } \\
\text { rectangular }\end{array}$ & 0.4 & 79 & $\begin{array}{l}\text { ppectra of forced harmonics } \\
\text { consist of 0.4Hz, 1.2Hz, } \\
\text { 2Hz, etc modes }\end{array}$ \\
\hline
\end{tabular}

As an example, the following gives the details of the case F_6_2. In this case, all generators are represented by classical model except for the generator 79 which is in the round generator model, denoted as GENROU in PSS/E model library. Small signal analysis shows that all natural modes have reasonably good damping. A forced oscillation is injected into the excitation system of generator 79 as a rectangular-wave with a fundamental frequency at $0.2 \mathrm{~Hz}$ as shown in Fig.3. The rotor angle responses of the system are 
shown in Fig.4 where many generators across the system have comparable amplitude of oscillations. Amplitudes of excited harmonics are significant but different for different generators, which makes it difficult to identify the source of oscillations by direct observation on these curves.

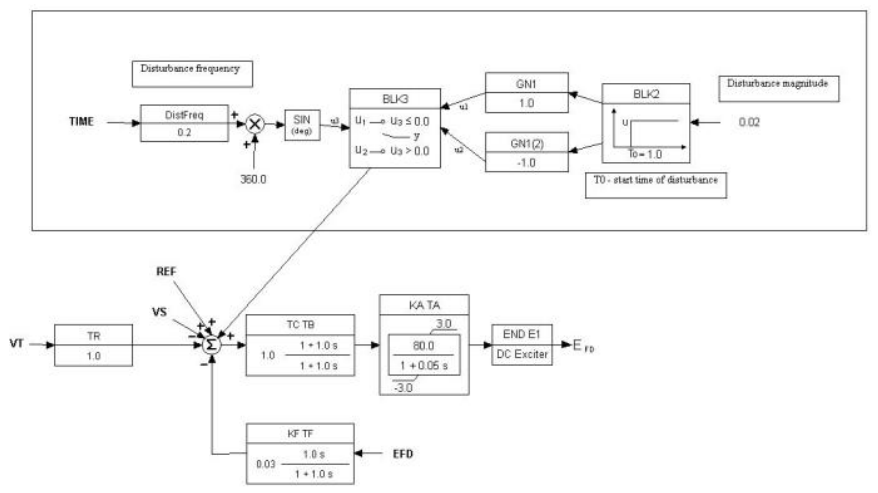

Fig. 3. Excitation system with injected rectangular-wave disturbance

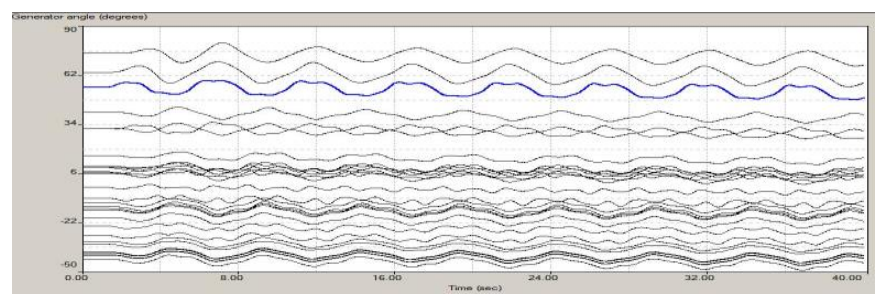

Fig. 4. Rotor angle responses in case F_6_2.

\section{ILLUSTRATIVE EXAMPLES OF USING TEST CASES ON THREE SOURCE LOCATION METHODS}

Three popular oscillation source location methods are tested using the whole library. Here we only present two cases for each method such that the method performs well on one case but poorly or even fails on the other one. The three methods under test are the damping torque based method [14], the estimated mode shape based method [6] and the dissipation energy flow based method [9].

\section{A. Damping torque based method}

The damping torque concept was initially proposed for single machine systems [16] and then extended to multimachine systems [14]. The damping torque based source location method requires measurements of rotor angle, rotor speed and electrical torque of generators and estimates the damping torque $K_{m d}$ for each single generator and for the dominant mode $m$. The generator with a negative damping torque coefficient is believed to provide negative contribution to the system damping so that it is identified as the source.

Poorly damped oscillation case PD_1 and forced oscillation case $F_{-} 1$ are used to test this method and the test results are shown in TABLE III. The results are summarized using two parameters: the generator number and the damping torque $K_{m d}$. In the case PD_1, the actual source G45 with negative damping torque coefficient at -1.81 is correctly captured by this method and all other generators have positive damping torque coefficients ranging from 0.91 to 7.89 . In the case $F_{-} 1$, the damping torque coefficients for all generators are found to be positive numbers ranging from 1.88 to 3.98 which means all generators are contributing positive damping and no generator is contributing negative damping to the oscillation therefore no source is found by this method.

TABLE III ESTIMATED DAMPING TORQUE COEFFICIENTS IN TwO SELECTED CASES

\begin{tabular}{lcc}
\hline \multirow{2}{*}{ Case \# } & \multicolumn{2}{c}{ (Generator, $\left.K_{m d}\right)$} \\
\cline { 2 - 3 } & Identified source generator(s) & Non-source generator(s) \\
\hline PD_1 & $(\mathrm{G} 45,-1.81)$ & $\left(\mathrm{G}^{*}, 0.91 \sim 7.89\right)$ \\
$\mathrm{F}_{-} 1$ & No source found & (All generators, 1.88 3.98) \\
\hline $\mathrm{G}^{*}$ represents all generators in the system except for G45.
\end{tabular}

Damping torque based source location method may locate the source for poorly damped oscillation cases but may fail under forced oscillation cases. From a practical point of view, the method is expected to be able to locate the source of oscillations regardless of its nature.

\section{B. Estimated mode shape based method}

Mode shape represents the relative amplitude and phasing of the oscillation throughout the system. Its definition is based on the right eigenvectors of the state matrix from the linearized system model. For real power systems, an accurate mode shape estimation from the model is difficult to get due to the inaccuracies in modeling. Instead, this method uses mode shape estimation from PMU measurement assuming adequate observability of the system by PMU. The rationale behind the method was derived from a 2-machine system and was assumed to be extensible to arbitrary multi-machine systems [6]. The method selects two dominant group of coherently oscillating generators, calculates an average angle for each group, selects the leading group and assumes that the leading generator in the leading group is the source of the oscillation.

Mode shapes of poorly damped oscillations in the cases PD_1 and PD_2 are shown in Fig.5. In case PD_1, the source G45 is correctly identified. In case PD_2, the bus 32 is found to be the leading one in the leading group such that it is identified as the source. However, the actual source is G65, which is the leading one in the lagging group.

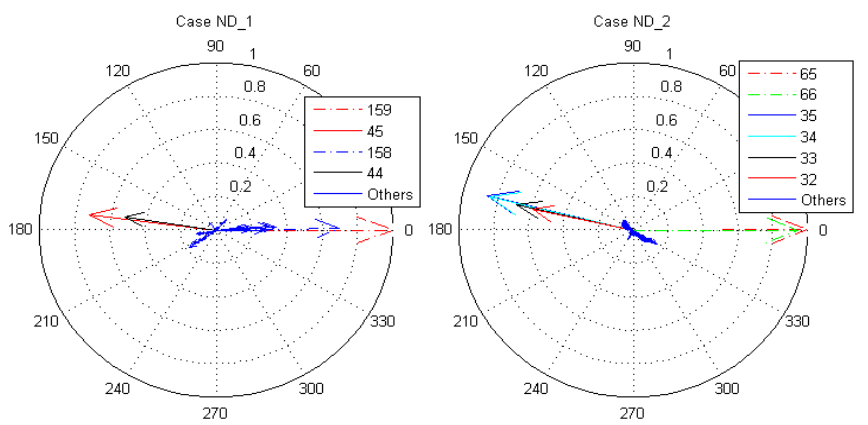

Fig. 5. Mode shape of bus angle in cases PD_1 and PD_2.

The method based on estimated mode shape [6] can correctly locate the source in many situations while sometimes 
providing wrong results. Application of this method also requires some heuristic assumptions, which makes it not easy to use as a robust universal method.

\section{Dissipation energy flow based method}

This method [9] utilizes the analytical description of dissipation energy flow in branches of power systems. Tracing the source of dissipation energy is equivalent to tracing the source of oscillations. Thus, the dissipation energy flow is first calculated for branches connecting each generator to the system. If the dissipation energy flow from a generator to the system is increasing over time, then that generator is the source of forced or undamped oscillations.

Poorly damped oscillation cases PD_1 and PD_6 are used for illustration and the results are shown in Fig.6 and Fig.7. In case PD_1, the source G45 is correctly identified since it has an increasing energy as shown in Fig.6. In case PD_6, which has two sources, the source G45 is correctly and clearly identified while the other source G159 does not have an increasing energy such that it may not be identified by this method. It is worth to mention that this method can correctly and uniquely identify the sources of all forced oscillation cases in the library.

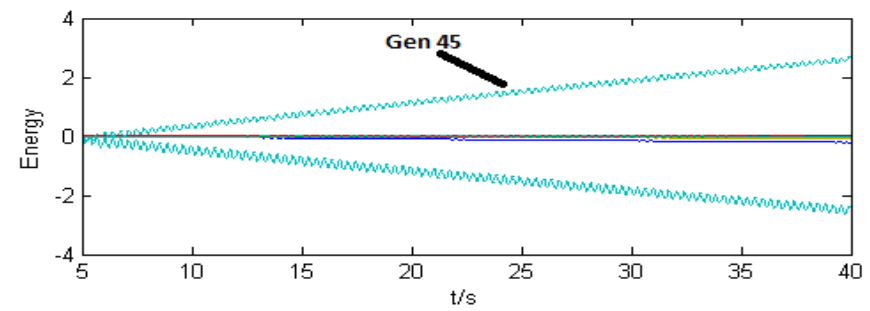

Fig. 6. Energy flowing out of generators in case PD_1.

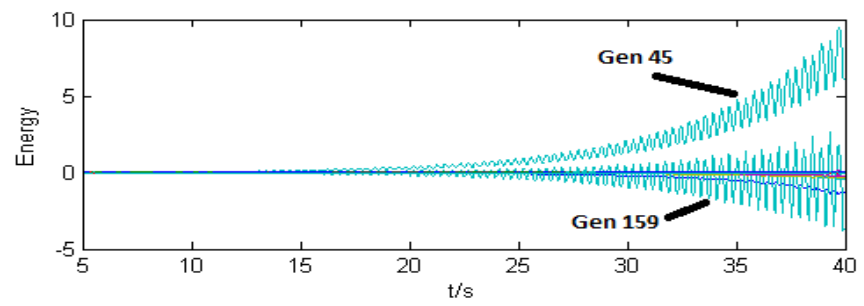

Fig. 7. Energy flowing out of generators in case PD_6.

In summary, the dissipation energy based method is capable of identifying the source of both poorly damped and forced oscillations. But it may fail to identify all oscillation sources in multiple-source oscillation cases, while the source with the largest negative contribution to the damping could be identified.

\section{CONCLUSIONS}

This paper presents the library of simulated oscillation cases designed to test methods for locating the source of poorly damped or forced oscillations. The cases in this library represent different situations that could possibly happen in real systems. The use of the library is illustrated with three popular source location methods and demonstrated that the variety of test cases are useful to reveal a potential weakness of a method under test. The purpose of this library is to help validate, evaluate and improve methods for locating the source of poorly damped or forced oscillations in power systems.

\section{REFERENCES}

[1] Zhang, Q.F.; Luo, X.; Litvinov, E.; Dahal, N.; Parashar, M.; Hay, K.; Wilson, D., "Advanced grid event analysis at ISO New England using PhasorPoint," IEEE PES General Meeting, National Harbor, MD, 27$31 \mathrm{Jul} 2014$

[2] Zhang, Q.F; Luo, X.; Maslennikov, S.; Ma, F.; Bertagnolli, D.; Ning, J; Ashwal, N.A.; Hay, K., "Oscillation source location and long term dynamic performance baselining study using ISO-NE's synchrophasor data," NASPI Working Group Meeting, San Mateo, CA, 23-24 Mar 2015

[3] ISO New England, "Synchrophasor infrastructure and data utilization in the ISO New England transmission region," Final Project Description in Smart Grid Investment Grant in 2009 American Recovery and Reinvestment Act, Apr 2015

[4] Dong, Q.; Zhang, L.; Yan, X.; Liu, X., "Automatic locating source method of compelled resonance low frequency oscillation in power grid," Proceedings of the CSEE, vol.32, no.28, pp68-75, Oct 2012

[5] Gao, Y.; Liu, D.; Huang, G.; Shi, Q., "Locating method of disturbance source of forced power oscillation based on prony anyasis," Electricity Distribution (CICED), China International Conference on, Shanghai, 10-14 Sept. 2012

[6] Ashwal, N. A.; Wilson, D.; Parashar, M., "Identifying sources of oscillations using wide area measurements," Grid of the Future Symposium, CIGRE USNC, Houston, TX, 19-21 Oct 2014

[7] Sarmadi, S.A.N.; Venkatasubramanian, V., "Inter-Area Resonance in Power Systems From Forced Oscillations," IEEE Trans. Power Syst., vol.PP, no.99, pp.1-9, Feb 2015

[8] Li, Y.; Shen, C.; Liu, F., "An energy-based methodology for locating the source of forced oscillations in power systems," in Power System Technology (POWERCON), IEEE International Conference on, Oct.30-Nov.2 2012

[9] Chen, L.; Min, Y.; Hu, W., "An energy-based method for location of power system oscillation source," IEEE Trans. Power Syst., vol.28, no.2, pp.828-836, May 2013

[10] Yang, Y.; Liu, T.; Li, X.; Hu, N.; Shi, H., "An equivalent circuit approach to locate source of power system forced power oscillation," Power System Technology, vol.36, no.11, pp101-108, Nov 2012

[11] Ma, J.; Zhang, P.; Fu, H.; Bo, B.; Dong, Z., "Application of Phasor Measurement Unit on Locating Disturbance Source for Low-Frequency Oscillation," IEEE Trans. Smart Grid, vol.1, no.3, pp.340-346, Dec. 2010

[12] C. Jiang, J. Liu, Y. Liu, J. Gou, C. Chen, R. Chen, M. Bazargan, "Online forced oscillation detection and identification based on wide area measurement system and CELL theory," Electric Power Automation Equipment, vol.35, no.2, pp125-132, Feb. 2015

[13] Nudell, T.R.; Chakrabortty, A., "Graph-Theoretic Methods for Measurement-Based Input Localization in Large Networked Dynamic Systems," IEEE Trans. Autom. Control, vol.60, no.8, pp.2114-2128, Aug 2015

[14] Y. Li, Y. Huang, J. Liu, W. Yao, J. Wen, "Power system oscillation source location based on damping torque analysis," Power System Protection and Control, vol.43, no.14, pp.84-91, Jul 2015

[15] Test Cases Library of Sustained Power System Oscillations [Online] Available: http://web.eecs.utk.edu/ kaisun/Oscillation/

[16] Demello, F.P.; Concordia, C., "Concepts of Synchronous Machine Stability as Affected by Excitation Control," IEEE Trans. Power App. Syst., vol.PAS-88, no.4, pp.316-329, Apr 1969 